\title{
ISOLATED ORBITS OF THE ADJOINT ACTION AND AREA-MINIMIZING CONES
}

\author{
MICHAEL KERCKHOVE
}

(Communicated by Peter Li)

\begin{abstract}
Using a criterion of Lawlor, it is shown that the cone over an isolated orbit of the adjoint action of $\mathrm{SU}(n)$ on the unit sphere in the vector space of traceless $n$-by- $n$ Hermitian symmetric matrices is area-minimizing for $n>2$. Likewise, the cone over an isolated orbit of the adjoint action of $\operatorname{SO}(n)$ is shown to be area-minimizing for $n>3$.
\end{abstract}

\section{INTRODUCTION}

Minimal cones over subsets of the unit sphere are candidates for area-minimizing surfaces with singularities. Recently, Lawlor [L] has developed a criterion for determining whether such a minimal cone is indeed area-minimizing. In this paper, Lawlor's criterion is applied to cones over certain orbits of the adjoint action of $\mathrm{SU}(n)$, respectively $\mathrm{SO}(n)$, in the vector space of Hermitian symmetric, respectively real symmetric, matrices. In its simplest form, Lawlor's criterion states that if the "vanishing angle" exists at each point of the cone and is less than half the "normal radius", then the cone is area-minimizing. Requisite terminology is discussed in $\S 2$. Normal radii and shape operators are computed in $\S 3$, and the main theorems are proved in $\S 4$.

\section{NotATION AND TERMINOLOGY}

The vector space $\mathscr{H}_{n}$ of traceless $n$-by- $n$ Hermitian symmetric matrices has a natural Euclidean metric given by

$$
\langle\boldsymbol{\alpha}, \boldsymbol{\beta}\rangle=\frac{1}{2} \operatorname{Trace}\left(\boldsymbol{\alpha} \overline{\boldsymbol{\beta}}^{t}\right) .
$$

The left action of $\mathrm{SU}(n)$ on $\mathscr{X}_{n}$ given by

$$
g \cdot \boldsymbol{\alpha}=g \boldsymbol{\alpha} \bar{g}^{t}, \quad g \in \mathrm{SU}(n), \alpha \in \mathscr{H}_{n},
$$

Received by the editors September 18, 1992.

1991 Mathematics Subject Classification. Primary 49Q05, 53C42.

Key words and phrases. Area-minimizing surface, cone. 
is clearly isometric. Under the action each $\mathrm{SU}(n)$-orbit intersects the set

$$
\Lambda_{n}=\left\{\lambda=\operatorname{diag}\left(\lambda_{1}, \ldots, \lambda_{n}\right) \mid \lambda_{j} \in \mathbf{R}, \lambda_{1} \geq \cdots \geq \lambda_{n}, \sum_{j=1}^{n} \lambda_{j}=0\right\} \subset \mathscr{H}_{n}
$$

in a unique point, the diagonal representative of the orbit. Orbit representatives can be further distinguished according to the multiplicities of their distinct eigenvalues. For representatives of principal orbits, each eigenvalue has multiplicity one. Singular orbits have repeated eigenvalues. The most singular orbits (discounting the origin) have only two distinct eigenvalues. If the diagonal matrix $\lambda \in \Lambda_{n}$ has length 1 , its $\mathrm{SU}(n)$-orbit will be a submanifold of the unit sphere in $\mathscr{\ell}_{n}$. On the unit sphere, orbits with only two distinct eigenvalues are isolated; there can be no continuous families of orbits of this type since the two conditions Trace $\lambda=0$ and $|\lambda|=1$ completely determine the two eigenvalues once the multiplicities are known. Cones over such isolated orbits are minimal but not necessarily area-minimizing.

For other $\mathrm{SU}(n)$-orbit types on the unit sphere, minimal cones correspond to orbits of maximum volume. The $\Lambda_{n}$ representative for the maximum volume orbit in the orbit type having $k$ distinct eigenvalues $\lambda_{1}, \ldots, \lambda_{k}$ of multiplicities $m_{1}, \ldots, m_{k}$ is a solution to the following problem: maximize the product

$$
\prod_{1 \leq i \leq j \leq k}\left(\lambda_{i}-\lambda_{j}\right)^{m_{i} m_{j}} \quad \text { subject to } \sum_{i=1}^{k} m_{i} \lambda_{i}=0 \text { and } \frac{1}{2} \sum_{i=1}^{k} m_{i} \lambda_{i}^{2}=1 .
$$

Though simple to state, this problem appears difficult to solve. Solutions for $n=3$ and 4 may be found in Kerckhove [K].

Remarks in the previous paragraphs remain valid if $\mathscr{H}_{n}$ is replaced by its real analog, the vector space $\mathscr{S}_{n}$ of traceless $n$-by- $n$ real symmetric matrices and the special unitary group $\mathrm{SU}(n)$ is replaced by the special orthogonal group $\mathrm{SO}(n)$.

More generally, the cone $C$ over a submanifold $B$ of the unit sphere is the union of open rays from the origin through each point of $B$. Such a cone may be orientable or nonorientable. A cone is said to be area-minimizing if it has least area among all surfaces (integral currents, resp. integral currents modulo 2 ) with boundary $B$. A simple sufficient test for area-minimization is stated as Theorem 1.3.5 of Lawlor [L]. To apply the test to a particular cone, two geometric quantities must be computed: the maximum value of the Euclidean norm of the shape operator $\max _{q}\left(\sup _{\nu}\left\|A_{\nu}\right\|\right)$ as $\nu$ ranges over all unit normals to $C$ at a point $q$ and $q$ ranges over $B$, and the minimum value of the "normal radius" at $q$, again as $q$ ranges over $B$. The normal radius at $q$ is computed as follows: For each arc of a great circle passing through $q$ normal to $B$ and intersecting $B$ only at $q$ and at its two end points, compute the angles subtended by line segments joining the origin to $q$ and to each of the end points, take the smaller of these two angles, and then minimize this angle over all such great circles through $q$. Generally speaking, a cone with small curvature, as measured by the Euclidean norms of its shape operators, and large normal radius will be area-minimizing. To prove this, Lawlor constructs an areanonincreasing retraction from the ambient Euclidean space onto the cone which 
leaves the cone pointwise fixed. The retraction is constructed separately on the normal space to each ray of the cone, provided the shape operator norms along the ray are small enough. The inverse image of an open ray of the cone under this retraction is an open, solid cone of revolution lying in the normal space to the cone along the original ray. The angle between the original ray and the boundary of this solid cone is called the "vanishing angle" at the point $q$ of $B$ that determines the original ray. The vanishing angle at $q$ may be estimated in terms of the dimension of $C$ and the maximum shape operator norm. In order for the retraction to be well defined, no two of these solid cones of revolution can intersect; thus the vanishing angle must be sufficiently small. Lawlor's criterion cited above states that if, at each point of $B$, the vanishing angle is less than half the normal radius, then the cone over $B$ is area-minimizing. See Chapter 1 of $[\mathrm{L}]$ for a more complete development of these ideas.

\section{SHAPE OPERATORS AND NORMAL RADII FOR ORBITS HAVING TWO DISTINCT EIGENVALUES}

To compute shape operators and normal radii, it is convenient to introduce matrices $S_{j k}$ and $Q_{j k}$ defined by

$$
S_{j k}(l m)=\delta_{j l} \delta_{k m}+\delta_{j m} \delta_{k l}, \quad Q_{j k}(l m)=\delta_{j l} \delta_{k m}-\delta_{j m} \delta_{k l} .
$$

The matrices $S_{j k}, 1 \leq j \leq k \leq n$, and $i Q_{j k}, 1 \leq j<k \leq n$, form an orthogonal basis for the vector space of all Hermitian symmetric $n$-by- $n$ matrices, while the skew-Hermitian matrices $i S_{j k}$ and $Q_{j k}$ span the tangent space to $\mathrm{SU}(n)$ at the identity.

Suppose now that $\lambda \in \Lambda_{n}$ is a traceless diagonal matrix of length 1 having two distinct eigenvalues $\lambda_{1}>0>\lambda_{2}$ of multiplicities $m_{1}$ and $m_{2}$ respectively. The relations $m_{1}+m_{2}=n, m_{1} \lambda_{1}+m_{2} \lambda_{2}=0$, and $m_{1} \lambda_{1}^{2}+m_{2} \lambda_{2}^{2}=2$ yield explicit formulas for the eigenvalues

$$
\lambda_{1}=\sqrt{2 m_{2} / n m_{1}}, \quad \lambda_{2}=-\sqrt{2 m_{1} / n m_{2}} .
$$

On the unit sphere in $\mathscr{H}_{n}$ the orbit of $\lambda$ is isolated; hence, the cone over the orbit is minimal. The orbit of $\lambda$ is the image of $\mathrm{SU}(n)$ by the map $f_{\lambda}$ : $\mathrm{SU}(n) \rightarrow \mathscr{H}_{n}$ defined by $f_{\lambda}(g)=g \lambda \bar{g}^{t}$. The tangent space to the orbit at the diagonal representative $\lambda$ is spanned by the vectors

$$
\begin{aligned}
f_{\lambda^{*}} Q_{j k} & =\left[\lambda, Q_{j k}\right]=\left(\lambda_{j}-\lambda_{k}\right) S_{j k}, \\
f_{\lambda^{*}} i S_{j k} & =\left[\lambda, i S_{j k}\right]=\left(\lambda_{j}-\lambda_{k}\right) i Q_{j k}
\end{aligned}
$$

with $1 \leq j \leq m_{1}, m_{1}+1 \leq k \leq n$. Thus the cone over the orbit has dimension $2 m_{1} m_{2}+1$. The space of unit normals consists of Hermitian symmetric matrices

$$
\boldsymbol{\nu}=\left(\begin{array}{cccccc}
\nu_{11} & \cdots & \nu_{1 m} & 0 & \cdots & 0 \\
\vdots & & & & & \vdots \\
\nu_{m 1} & \cdots & \nu_{m m} & 0 & \cdots & 0 \\
0 & \cdots & 0 & \nu_{m+1 m+1} & \cdots & \nu_{m+1 n} \\
\vdots & & & & & \vdots \\
0 & \cdots & 0 & \nu_{n m+1} & \cdots & \nu_{n n}
\end{array}\right)=\left(\begin{array}{cc}
\nu_{1} & 0 \\
0 & \nu_{2}
\end{array}\right)
$$


satisfying $|\boldsymbol{\nu}|^{2}=\left|\nu_{1}\right|^{2}+\left|\nu_{2}\right|^{2}=1$, Trace $\nu=$ Trace $\nu_{1}+$ Trace $\nu_{2}=0$, and $\operatorname{Trace}(\nu \lambda)=\lambda_{1} \operatorname{Trace} \nu_{1}+\lambda_{2} \operatorname{Trace} \nu_{2}=0$. The last two conditions imply that Trace $\nu_{1}=$ Trace $\nu_{2}=0$. In particular, if $m_{j}=1$, then $\nu_{j}=0$. Restricting to matrices whose entries are real, it is clear that the cone over the $\mathrm{SO}(n)$-orbit of $\lambda$ in $\mathscr{S}_{n}$ has dimension $m_{1} m_{2}+1$, with unit normals of the same form as above.

Proposition 1. Let $\lambda$ be a traceless diagonal matrix having two distinct eigenvalues $\lambda_{1}>0>\lambda_{2}$ of multiplicities $m_{1}$ and $m_{2}$ respectively. Then the normal radius for the cone over both the $\mathrm{SU}(n)$-orbit and the $\mathrm{SO}(n)$-orbit of $\lambda$ is $\cos ^{-1}\left(1-\left(m_{1}+m_{2}\right) / m_{1} m_{2}\right)$.

Proof. For any normal vector $\nu$ to the cone at its length 1 diagonal orbit representative $\lambda$, the block diagonal form of $\nu$ implies that the Lie bracket $[\nu, \lambda]=0$. Hence $\boldsymbol{\nu}$ and $\lambda$ are simultaneously diagonalizable via an appropriate unitary, respectively orthogonal, change of basis matrix $b$ which is also block diagonal.

Suppose the normal geodesic $\cos \phi \lambda+\sin \phi \nu$ intersects the orbit of $\lambda$ at a point $\mu=g \lambda \bar{g}^{t}$ for some $g \in \mathrm{SU}(n)$, respectively $\mathrm{SO}(n)$, so that, for some fixed angle $\phi$,

$$
\cos \phi \lambda+\sin \phi \nu=g \lambda \bar{g}^{t} .
$$

Applying the change of basis matrix $b$ yields

$$
\cos \phi b \lambda \bar{b}^{t}+\sin \phi b \nu \bar{b}^{t}=b g \lambda \overline{\lambda(b g)}^{t} .
$$

Since $b \lambda \bar{b}^{t}=\lambda$, the left-hand side of this expression is clearly diagonal. Moreover, such a change of basis is isometric. Thus the normal radius at $\lambda$ may be computed using only diagonal matrices. By homogeneity, the normal radius for the cone is equal to the smallest nonzero angle between diagonal matrices in the same orbit. This minimum angle is achieved by interchanging a single pair of unequal eigenvalues. The resulting angle satisfies $\cos \phi=$ $1-\left(m_{1}+m_{2}\right) / m_{1} m_{2}$.

To compute shape operator norms, extend each unit normal vector to the cone at $\lambda$ to an invariant unit normal on a neighborhood of $\lambda$ by setting

$$
\boldsymbol{\nu}\left(g c \lambda \bar{g}^{t}\right)=g \nu \bar{g}^{t},
$$

where $c \in \mathbf{R}^{+}$and $g \in \mathrm{SU}(n)$ is near the identity. Then $A_{\nu} \lambda=0$, while, for $\boldsymbol{\tau} \in \operatorname{Span}_{\mathbf{R}}\left\{Q_{j k}, i S_{j k} \mid 1 \leq j \leq m_{1}, m_{1} \leq k \leq n\right\}$,

$$
A_{\nu} f_{\lambda^{*}} \tau=-\nabla_{f_{\lambda^{*} \tau} \nu}=-[\boldsymbol{\nu}, \boldsymbol{\tau}] .
$$

As a consequence of the following lemma, the maximum shape operator norm may be determined using only diagonal unit normals at $\lambda$.

Lemma 1. Let $\lambda \in \Lambda_{n}$ be a traceless diagonal $n$-by- $n$ matrix having two distinct eigenvalues $\lambda_{1}>0>\lambda_{2}$ of multiplicities $m_{1}$ and $m_{2}$ respectively. Let $\nu$ be an invariant unit normal to the cone over the $\mathrm{SU}(n)$-orbit of $\lambda$, and let $b$ be an element of the isotropy subgroup at $\lambda$. Then the shape operator norms $\left\|A_{b \cdot \nu}\right\|$ and $\left\|A_{\nu}\right\|$ are equal. 
Proof. The left action $b \cdot x=b x \bar{b}^{t}$ is a linear isometry of each of the following vector spaces: $\mathscr{H}_{n}$, leaving $\lambda$ fixed; the normal space at $\lambda$ to the cone over the orbit of $\lambda$; the tangent space at $\lambda$ to the cone over the orbit of $\lambda$; and the tangent space to $\mathrm{SU}(n)$ at the identity. The lemma may be proved by observing that

(1) For $1 \leq j \leq m_{1}<k \leq n, S_{j k}=\frac{1}{\lambda_{1}-\lambda_{2}} f_{\lambda^{*}} Q_{j k}$ while $i Q_{j k}=\frac{1}{\lambda_{1}-\lambda_{2}} f_{\lambda^{*}} i S_{j k}$.

(2) For $\tau \in \operatorname{Span}_{\mathbf{R}}\left\{Q_{j k}, i S_{j k} \mid 1 \leq j \leq m_{1}, m_{1} \leq k \leq n\right\}, b \cdot f_{\lambda^{*}} \tau=$ $f_{\lambda^{*}}(b \cdot \tau)$.

(3) $\left\|A_{b \cdot \nu}\left(b \cdot f_{\lambda^{*}} \tau\right)\right\|=\|b \cdot[\boldsymbol{\nu}, \boldsymbol{\tau}]\|=\|[\boldsymbol{\nu}, \boldsymbol{\tau}]\|=\left\|A_{\nu}\left(f_{\lambda^{*}} \boldsymbol{\tau}\right)\right\|$.

(4) $\left\|A_{b \cdot \nu}\right\|^{2}=\sum_{j=1}^{m_{1}} \sum_{k=m_{1}+1}^{n}\left\|A_{b \cdot \nu} b \cdot S_{j k}\right\|^{2}+\left\|A_{b \cdot \nu} b \cdot i Q_{j k}\right\|^{2}$.

The remaining details are left to the reader. A similar argument establishes the corresponding lemma when unitary groups are replaced by orthogonal groups.

Proposition 2. Let $\lambda$ be a traceless diagonal matrix having two distinct eigenvalues $\lambda_{1}>0>\lambda_{2}$ of multiplicities $m_{1}$ and $m_{2}$ respectively. Then the maximum shape operator norm for the cone over the $\mathrm{SU}(n)$-orbit of $\lambda$ satisfies

$$
\max _{\nu}\left\|A_{\nu}\right\|^{2}= \begin{cases}\frac{2 m_{1} m_{2}}{m_{1}+m_{2}} \max \left(m_{1}, m_{2}\right) & \text { if } 1 \neq m_{1}, m_{2}, \\ \frac{2(n-1)}{n} & \text { if } m_{1}=1 \text { or } m_{2}=1,\end{cases}
$$

while the maximum shape operator norm for the cone over the $\mathrm{SO}(n)$-orbit of $\lambda$ satisfies

$$
\max _{\nu}\left\|A_{\nu}\right\|^{2}= \begin{cases}\frac{m_{1} m_{2}}{m_{1}+m_{2}} \max \left(m_{1}, m_{2}\right) & \text { if } 1 \neq m_{1}, m_{2}, \\ \frac{(n-1)}{n} & \text { if } m_{1}=1 \text { or } m_{2}=1 .\end{cases}
$$

Proof. For a diagonal unit normal $\nu=\operatorname{diag}\left(\nu_{11}, \ldots, \nu_{n n}\right)$ at $\lambda$,

$$
\begin{aligned}
\left\|A_{\nu}\right\|^{2} & =\sum_{j=1}^{m_{1}} \sum_{k=m_{1}+1}^{n}\left\|A_{\nu} S_{j k}\right\|^{2}+\left\|A_{\nu} i Q_{j k}\right\|^{2} \\
& =\frac{2}{\left(\lambda_{1}-\lambda_{2}\right)^{2}} \sum_{j=1}^{m_{1}} \sum_{k=m_{1}+1}^{n}\left(\nu_{j j}-\nu_{k k}\right)^{2} \\
& =\frac{2}{\left(\lambda_{1}-\lambda_{2}\right)^{2}}\left(m_{2}\left\|\nu_{1}\right\|^{2}+m_{1}\left\|\nu_{2}\right\|^{2}-2 \text { Trace } \nu_{1} \text { Trace } \nu_{2}\right) \\
& =\frac{m_{1} m_{2}}{n}\left(m_{2}\left\|\nu_{1}\right\|^{2}+m_{1}\left\|\nu_{2}\right\|^{2}\right) .
\end{aligned}
$$

The formulas for the maximum shape operator norms follow easily from the fact that $|\boldsymbol{\nu}|^{2}=\frac{1}{2}\left(\left\|\nu_{1}\right\|^{2}+\left\|\nu_{2}\right\|^{2}\right)=1$.

\section{VANISHING ANGLES AND THE MAIN THEOREM}

It is now possible to estimate the vanishing angle for the cone over the orbit of $\lambda$. For cones of dimension at most 12 , estimated vanishing angles are recorded 
in the table in $\S 1.4$ of [L]. Estimates of vanishing angles $V_{C}\left(\operatorname{dim} C, \max \left\|A_{\nu}\right\|\right)$ for cones $C$ of dimension greater than 12 are made using the inequality

$$
V_{C}\left(\operatorname{dim} C, \max \left\|A_{\nu}\right\|\right)<\tan ^{-1}\left(\frac{12}{\operatorname{dim} C} \tan \left(V_{C}\left(12, \frac{12}{\operatorname{dim} C} \max \left\|A_{\nu}\right\|\right)\right)\right)
$$

that appears in Theorem 1.4.2 of [L].

If one of the multiplicities is 1 , the calculations are particularly simple. The cone over the orbit of $\lambda$ has dimension $2 n-1$. From the table in [L], one finds that for $3 \leq n \leq 6$ the vanishing angle is less than $24^{\circ}$ while the normal radius is at least $90^{\circ}$. Thus Theorem 1.3 .5 of [L] may be applied directly to show that the resulting cones are area-minimizing. Note that when $n=2$ the resulting cone is a hypersurface in $\mathscr{H}_{2}$ which cannot be area-minimizing; an area-minimizing hypersurface containing a singularity must have dimension at least 7 .

For $n>6$, the vanishing angle $V_{C}\left(2 n-1, \sqrt{\frac{2(n-1)}{n}}\right)$ satisfies

$$
\begin{aligned}
& V_{C}\left(2 n-1, \sqrt{\frac{2(n-1)}{n}}\right) \\
& \quad<\tan ^{-1}\left(\frac{12}{2 n-1} \tan \left(V_{C}\left(12, \frac{12}{2 n-1} \sqrt{\frac{2(n-1)}{n}}\right)\right)\right) \\
& \quad<V_{C}(12, \sqrt{2}) \approx 8.08^{\circ} .
\end{aligned}
$$

Clearly $V_{C}\left(2 n-1, \sqrt{\frac{2(n-1)}{n}}\right)<45^{\circ}$, and since the normal radius is at least $90^{\circ}$, the cone is area-minimizing.

When both multiplicities are greater than 1 , the cone dimension is greater than 12 except when $m_{1}=m_{2}=2$. In this case, the cone has dimension 9 and maximum shape operator norm 2 , resulting in a vanishing angle of $\approx 11.57^{\circ}$. The normal radius is $90^{\circ}$, so the cone is area-minimizing. When the cone dimension is greater than 12 , the vanishing angle

$$
V_{C}\left(2 m_{1} m_{2}+1, \sqrt{\frac{2 m_{1} m_{2}}{m_{1}+m_{2}} \max \left(m_{1}, m_{2}\right)}\right)
$$

satisfies

$$
\begin{aligned}
& V_{C}\left(2 m_{1} m_{2}+1, \sqrt{\frac{2 m_{1} m_{2}}{m_{1}+m_{2}}}\right) \\
& <\tan ^{-1}\left(\frac{12}{2 m_{1} m_{2}+1} \tan \left(V_{C}\left(12, \frac{12}{2 m_{1} m_{2}+1} \sqrt{\frac{2 m_{1} m_{2}}{m_{1}+m_{2}} \max \left(m_{1}, m_{2}\right)}\right)\right)\right) \\
& <\tan ^{-1}\left(\frac{12}{2 m_{1} m_{2}+1} \tan \left(V_{C}\left(12, \frac{12}{\sqrt{2\left(m_{1}+m_{2}\right) \min \left(m_{1}, m_{2}\right)}}\right)\right)\right)
\end{aligned}
$$




$$
\begin{aligned}
& <\tan ^{-1}\left(\frac{12}{2 m_{1} m_{2}+1} \tan \left(V_{C}\left(12, \frac{12}{2 \sqrt{5}}\right)\right)\right) \\
& <\tan ^{-1}\left(\frac{12}{2 m_{1} m_{2}+1} \tan \left(8.55^{\circ}\right)\right) \\
& <\tan ^{-1}\left(\frac{2}{2 m_{1} m_{2}+1}\right)<\frac{2}{2 m_{1} m_{2}+1}<\frac{\pi}{2\left(m_{1}+m_{2}\right)}=\frac{\pi}{2 n} .
\end{aligned}
$$

On the other hand, the normal radius for the cone satisfies

$$
\begin{aligned}
\text { normal radius } & =\cos ^{-1}\left(1-\frac{m_{1}+m_{2}}{m_{1} m_{2}}\right) \geq \cos ^{-1}\left(1-\frac{4}{m_{1}+m_{2}}\right) \\
& =\cos ^{-1}\left(1-\frac{4}{n}\right)>\frac{\pi}{n} .
\end{aligned}
$$

The last inequality follows from the fact that $\cos \left(\frac{\pi}{n}\right)>1-\frac{\pi^{2}}{2 n^{2}}>1-\frac{4}{n}$ when $n \geq 4$. These estimates establish the following theorem for cones over isolated $\mathrm{SU}(n)$-orbits. Similar estimates yield the corresponding theorem for cones over isolated orbits of the special orthogonal groups.

Theorem 1. Let $\mathrm{SU}(n)$ act on the unit sphere in $\mathscr{H}_{n}$ by conjugation. With the exception of the cone over the $\mathrm{SU}(2)$-orbit of $\operatorname{diag}(1,-1)$ in $\mathscr{H}_{2}$, every cone over an isolated $\mathrm{SU}(n)$-orbit is area-minimizing.

Theorem 2. Let $\mathrm{SO}(n)$ act on the unit sphere in $\mathscr{S}_{n}$ by conjugation. With the exception of the cones over the $\mathrm{SO}(2)$-orbit of $\operatorname{diag}(1,-1)$ in $\mathscr{I}_{2}$ and the $\mathrm{SO}(3)$ orbits of $\pm \frac{1}{\sqrt{3}} \operatorname{diag}(1,1,-2)$ in $\mathscr{S}_{3}$, every cone over an isolated $\mathrm{SO}(n)$-orbit is area-minimizing.

The exceptional cones over $\mathrm{SO}(3)$-orbits mentioned in the latter theorem have dimension 3 in $\mathscr{S}_{3}$, a vector space of dimension 5. Consult [Mh1] for the known area-minimizing properties of these unorientable cones, the cones over the Veronese surface, and its opposite. In [Mh2] and [K], a strategy for calibrating cones over orbits of the adjoint action is outlined.

\section{ACKNOWLEDGMENT}

The author wishes to thank Gary Lawlor for suggesting this problem.

\section{REFERENCES}

[K] M. Kerckhove, Minimal cones and closed differential forms on $\operatorname{SO}(n) \times \Lambda_{n}$, preprint.

[L] G. Lawlor, $A$ sufficient criterion for a cone to be area-minimizing, Mem. Amer. Math. Soc., vol. 91, no. 446, Amer. Math. Soc., Providence, RI, 1991.

[Mh1] T. Murdoch, Twisted calibrations, Trans. Amer. Math. Soc. 328 (1991), 239-257. Calibrations arising from the adjoint action of $\mathrm{SO}(n)$, preprint.

Department of Mathematics, University of Richmond, Richmond, Virginia 23173

E-mail address: mgk@mathcs .urich.edu 